\title{
The effect of residual thermal stresses on the fatigue crack growth of laser-annealed 304 stainless steels
}

\author{
L.W. Tsay ${ }^{\mathrm{a}, *}$, M.C. Young ${ }^{\mathrm{b}, 1}$, F.Y. Chou ${ }^{\mathrm{c}}$, R.K. Shiue $\mathrm{e}^{\mathrm{b}, 1}$ \\ ${ }^{a}$ Institute of Materials Engineering, National Taiwan Ocean University, Keelung 202, Taiwan, ROC \\ ${ }^{\mathrm{b}}$ Department of Materials Science and Engineering, National Taiwan University, Taipei 106, Taiwan, ROC \\ ${ }^{\mathrm{c}}$ Department of Materials Science and Engineering, National Dong Hwa University, Hualien 974, Taiwan, ROC
}

Received 28 April 2004; received in revised form 22 July 2004; accepted 22 July 2004

\begin{abstract}
The fatigue crack growth behavior of AISI 304 stainless steels annealed by a $\mathrm{CO}_{2}$ laser was investigated in the study. For the laser-annealed specimen prior to introducing a notch, the residual tensile stress was obtained around the center of the laser-annealed zone (LAZ), and the residual stress field changed gradually from tensile into compressive stress with increasing the distance away from the centerline of LAZ. Based on the experimental result, the laser-annealed specimen tested under low $\Delta K$ had a higher resistance to fatigue crack growth in the region preceding the LAZ after introducing a notch perpendicular to the LAZ. In contrast, the retardation of fatigue crack growth disappeared for the laser-annealed specimen stress-relieved at $850{ }^{\circ} \mathrm{C}$ for one hour. The presence of residual compressive stress field ahead of the crack tip resulted in the decreased fatigue crack growth rate (FCGR). Moreover, residual compressive stresses enhanced the effect of crack closure, and led to the formation of rubbed marks on the fatigue-fractured surface. As the fatigue crack propagated further into the LAZ, the residual tensile stress field ahead of crack tip did not accelerate the crack growth. The results indicated that tensile stress field ahead of the crack tip had little effect on the FCGR of the ductile 304 stainless steel.
\end{abstract}

(C) 2004 Elsevier B.V. All rights reserved.

Keywords: Laser annealing; Fatigue crack growth; Residual thermal stresses; AISI 304 stainless steels

\section{Introduction}

Residual stresses are usually developed as a result of plastic deformation and/or temperature gradient in the material. It is well known that residual stresses have a great influence on the performance of most structural alloys. Considerable efforts including both theoretical and experimental studies have been concentrated on the effect of residual stresses on fatigue properties of many engineering alloys [1-6]. It has been reported that the residual tensile stress accelerates the fatigue crack growth rate (FCGR) due to the enhancement of crack opening $[7,8]$. In contrast, the residual compressive stress can effectively retard the fatigue crack growth [5,9-12]. There-

\footnotetext{
* Corresponding author. Tel.: +886-2-246221926405; fax: +886-2-24625324

E-mail address: bo186@mail.ntou.edu.tw (L.W. Tsay).

${ }^{1}$ Fax: +886223634562.
}

fore, the fatigue life of many structural alloys can be greatly improved by the presence of residual compressive stresses $[5,6]$. Not only $\mathrm{d} a / \mathrm{d} N$ decreases but also the threshold of $\Delta K$ $(\Delta K$ th) increases with increasing the residual compressive stress.

Several models based on the initial distribution of residual stresses have been proposed to predict the influence of residual stresses on FCGR $[4,5,9]$. However, the computation of residual stress intensity using the weight function method may overestimate the effect of residual stresses on fatigue life for most alloys, if the redistribution of residual stresses is not considered simultaneously during the fatigue crack growth. The redistribution of residual stresses takes place during the fatigue crack growth, so the residual stress intensity factor $\left(K_{\text {res }}\right)$ in front of crack tip changes with crack propagation $[6,13]$. Investigation of the residual stress effect on the fatigue crack growth must also include the redistribution of residual stresses ahead of the crack tip during the fatigue crack growth 
instead of considering its initial stress state only. In general, the AISI 304 stainless steel (304SS) does not experience substantial phase transformation during the heat treatment, so the effect of microstructure on the FCGR is not considered. It is quite suitable to choose 304SS in evaluating the influence of residual thermal stresses on its FCGR.

The use of hole-drilling strain gage method to measure the residual stress in various regions of a weld is fairly difficult because of the irregular shape and/or discontinuity in the fusion zone. On the other hand, the application of laser surface treatment, e.g. laser annealing, can impose residual thermal stresses without changing the surface morphology of the specimen. Therefore, measurements of residual thermal stresses in the laser-annealed specimen are much easier than those in the weld. The purpose of this research is to evaluate the effect of residual thermal stresses, especially stress redistribution ahead of the fatigue crack tip, on the FCGR of the laser-annealed 304SS plate.

\section{Material and experimental procedures}

The chemical composition of 304SS plate was $18.03 \mathrm{wt} . \%$ Cr, 8.38 wt. $\%$ Ni, 0.043 wt.\% C, 1.03 wt.\% Mn, 0.46 wt.\% $\mathrm{Si}, 0.036$ wt. \% P, 0.005 wt. \% S, 0.45 wt. \% Cu and Fe balance. Laser surface treatments were carried out by using a Rofin-Sinar $5 \mathrm{~kW} \mathrm{CO}$ laser. The laser beam size on the specimen was about $6 \mathrm{~mm} \times 25 \mathrm{~mm}$ by using a rectangular lens to provide a uniform energy intensity within the treated area. Prior to laser treatments, the specimens with the dimensions of $250 \mathrm{~mm} L \times 120 \mathrm{~mm} W \times 4.6 \mathrm{~mm} T$ were sprayed with a black paint to enhance surface absorption of laser energy. The laser power and its scan rate were $2000 \mathrm{~W}$ and $1500 \mathrm{~mm} \mathrm{~min}^{-1}$. Symmetrical laser irradiations on both top and bottom sides of the specimen's surfaces at the specific location were performed in order to reduce distortion of the specimen after laser annealing.

Fig. 1 shows the schematic diagram of compact tension (CT) specimen used in the fatigue crack growth test. The

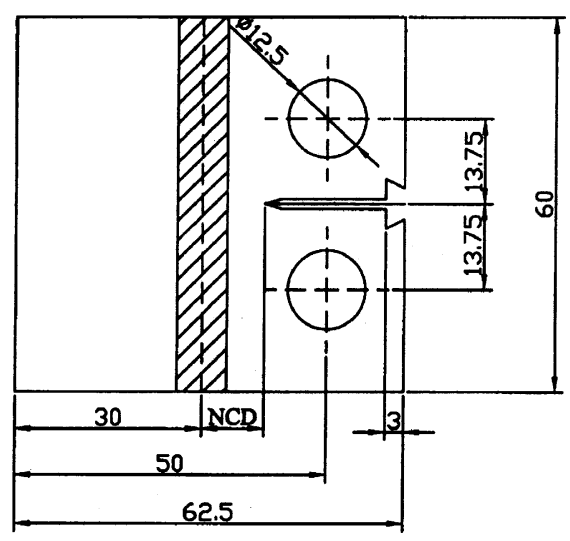

Fig. 1. The diagram of NL specimen used in the experiment, dimension in $\mathrm{mm}$ (NCD: the distance between the notch tip and centerline of LAZ). shaded area in the figure is the laser-annealed zone (LAZ). The width of LAZ is simply estimated by the laser beam scanned zone. Additionally, the NCD stands for the distance between the notch tip to centerline of LAZ. For the specimen with the crack growth direction normal to the laser scan direction, such a specimen is designated as the NL specimen throughout the experiment. An MTS equipped with a servo-controlled hydraulic testing system was applied in the fatigue test. The testing software 759.40 was capable of offering various testing conditions, including: constant load, constant $\Delta K$ and $K$-controlled tests. The compliance function proposed by Saxena and Hudak [14] was used to determine the fatigue crack length. Additionally, the crack length measured by crack-opening displacement (COD) gage was calibrated using a $30 \times$ traveling microscope.

In order to determine the effect of stress relaxation on FCGR of the NL specimen, the notch tip to centerline of LAZ distance (NCD) was preset at selected lengths, e.g. 10, 7.5 and $5 \mathrm{~mm}$. For instance, the NL-10 specimen represented that the notch tip was located at $10 \mathrm{~mm}$ away from the centerline of LAZ. In order to minimize the machining-induced stresses on the laser-annealed CT specimens, all CT specimens were cut from laser-annealed steel plate by a computercontrolled electro-discharged wire cutter with accurately controlled NCDs. Prior to the fatigue crack growth test, all CT specimens were pre-cracked $2 \mathrm{~mm}$ depth ahead the notch tip using the MTS machine. In the fatigue test, the loading frequency was $20 \mathrm{~Hz}$ with a constant amplitude sinusoidal waveform, and the applied stress ratio $(R)$ was kept at 0.1 throughout the experiment. At least three tests were performed for each testing condition. The fractured surface was examined using a Hitachi 4100 field emission scanning electron microscope (FESEM) with an operating voltage of $15 \mathrm{kV}$.

Measurements of the residual stress were performed using the hole-drilling strain gage method based on ASTM E837-92 specification. The strain-gage rosettes (CEA-06-062UL-120 manufactured by Measurements Group, Inc.) were attached at the centerline of the LAZ as well as various locations away from the centerline. The measured stress in the laser-annealed specimen was divided into longitudinal and transverse residual stresses with respect to laser scanning direction. Because the effect of residual stresses perpendicular to the notch tip was more prominent than those parallel to the notch tip in the fatigue test, only residual stresses perpendicular to crack growth direction were taken into consideration in the study.

\section{Results and discussion}

Fig. 2 shows the experimentally determined longitudinal residual thermal stress distribution in a laser-annealed steel plate prior to introducing a notch. It is noted that the longitudinal residual stresses are aligned normal to the crack propagation plane of the NL specimen. Based on the experimental observations, the longitudinal tensile stress field is observed around the LAZ. Increasing the distance away from the 


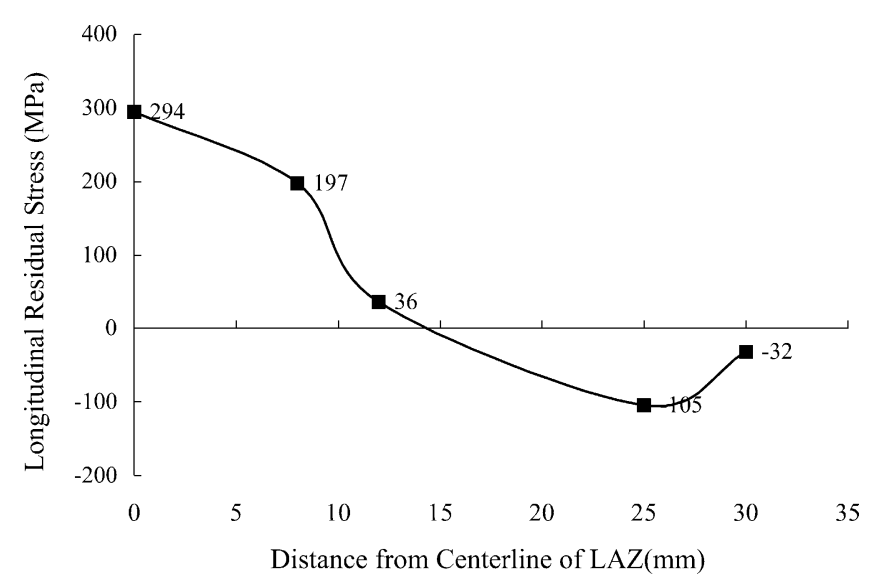

Fig. 2. Experimentally determined longitudinal residual stress profile in a laser-annealed steel plate prior to introducing a notch.

centerline of LAZ, the residual tensile stress is decreased and even converted from tensile into compressive stress. Meanwhile, the induced residual tensile stress of 304SS plate has to be self-balanced by the residual compressive stress in the remaining part of the specimen.

In order to realize the effect of an introduced notch upon the redistribution of residual thermal stresses, laser-annealed steel plate with various NCDs were made using an electrowire cutter. A hole with $1.6 \mathrm{~mm}$ in diameter was drilled at $2 \mathrm{~mm}$ ahead of the notch tip in order to measure the stress state of the notch tip. It was found that the longitudinal residual thermal stresses were $-28 \mathrm{MPa}$ for the specimen with $10 \mathrm{~mm} \mathrm{NCD}$ and $-49 \mathrm{MPa}$ for the specimen with $7.5 \mathrm{~mm}$ NCD. Based on Fig. 2, residual tensile stresses were available at the locations with 7.5 or $10 \mathrm{~mm}$ away from the centerline of LAZ prior to introducing notches. However, they were changed into compressive residual stresses after introducing notches in the above laser-annealed steel plates. The redistribution of stresses in the laser-annealed steel plate occurred drastically after introducing a notch into the specimen. Additionally, current experimental data behave the similar tendency as the previous results assisted by a finite element method [15]. Consequently, it is demonstrated that significant redistribution of the residual stress ahead of the crack tip proceeds with an introduced notch or crack. The redistribution of residual stresses may impose a great impact on the behavior of fatigue crack growth.

Fig. 3 displays the relation between $\mathrm{d} a / \mathrm{d} N$ and $\Delta K$ for 304SS base plate and laser-annealed specimens with the notch perpendicular to LAZ. The experimental result indicates that the FCGR of NL-10 specimen is much more sensitive to the change of $\Delta K$ than that of base plate. For the testing of NL-10 specimen, the initial $\Delta K$ value had to be raised above a critical value, or the stable crack growth of NL-10 specimen could not be obtained. Compared with the 304SS base plate, a threshold growth behavior of the NL-10 specimen occurred due to the existence of residual compressive stress field ahead of the notch tip. It is also noted that the FCGR of the NL-10 specimen increases rapidly with increas-

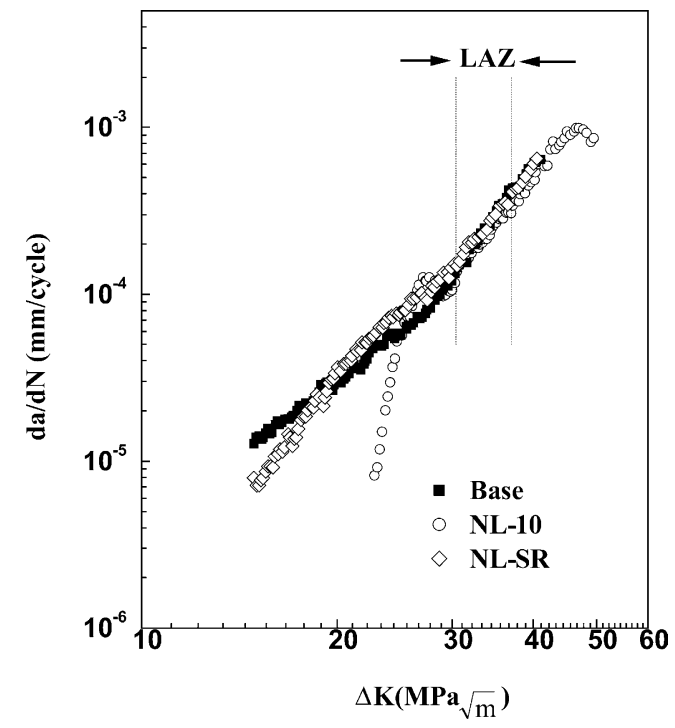

Fig. 3. The FCGR vs. $\Delta K$ curves for NL-10 and NL-SR specimens in comparison with the base metal.

ing $\Delta K$ once exceeding a critical value. The FCGR speeds up and eventually leads to a higher FCGR than that of the base plate in the region ahead of LAZ. As the crack propagates into the LAZ, similar FCGR between the NL-10 and base metal are observed. The significant difference in FCGR between NL-10 specimen and base metal in low $\Delta K$ range cannot be attributed to the microstructural effect. Actually, the redistribution of the residual thermal stress ahead of the crack tip is primarily responsible for the improved resistance to crack growth for the laser-annealed specimen.

If the stress-relief heat treatment is imposed on the laserannealed specimen at $850^{\circ} \mathrm{C}$ for $1 \mathrm{~h}$, i.e. NL-SR specimen shown in Fig. 3, the obvious retardation of crack growth in the low $\Delta K$ range disappears. In general, the NL-SR specimen exhibits similar FCGR as the base metal. The reduced FCGR in a weld have been reported previously [16-18]. The superior resistance to crack propagation in the weld metal and heat-affected zone as compared with that of base plate was observed in both the as-welded A514 and A36 steel weld $[16,17]$. However, the considerable increase in FCGR was observed for the welds subjected to a post-weld heat treatment $[16,18]$. It is in accordance with the above experimental result.

Fatigue crack growth tests were progressively performed on the laser-annealed specimens with different NCDs. Fig. 4 shows the relation between $\mathrm{d} a / \mathrm{d} N$ and $\Delta K$ for the laserannealed CT specimens with 5 and $7.5 \mathrm{~mm}$ NCDs in comparison with those of the base metal. It is obvious that further decreasing NCD deteriorates the initially high resistance to the fatigue crack growth of the laser-annealed specimen in low $\Delta K$ region. The discrepancy of the FCGR between laser-annealed specimens and the base metal in the low $\Delta K$ range disappears for specimens with shorter NCDs. The NL-5 specimen eventually exhibits the similar fatigue crack growth behavior as the base plate. 


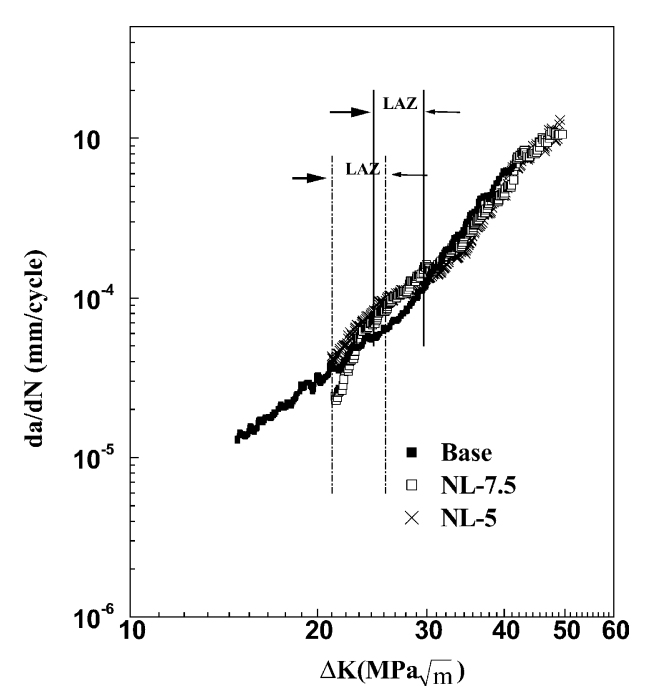

Fig. 4. The effect of various NCDs on the $\mathrm{d} a / \mathrm{d} N$ vs. $\Delta K$ curves of the laserannealed CT specimens in comparison with the base metal.

The effect of stress redistribution in the laser-annealed CT specimen during the fatigue crack growth was accessed by measuring residual stresses in both NL-10 and NL-7.5 specimens. The result further confirmed that the residual tensile stress was found within the LAZ, and the residual compressive stress was located ahead of the crack tip in both specimens. It has been studied previously that introducing a deep crack into the laser-annealed CT specimen causes a significant redistribution of residual stresses ahead the crack tip during the fatigue crack growth test [15]. Accordingly, current experimental measurements are well consistent with the computer simulated results [15]. Therefore, lower FCGR of NL-10 and NL-7.5 specimens in the low $\Delta K$ regime were primarily resulted from the residual compressive stress field ahead of the crack tip.

In order to magnify the change of FCGR in various regions of the NL-10 specimens, constant $\Delta K$ tests were performed at an $R$ ratio of 0.1 . Fig. 5 illustrates the crack propagating normal to the LAZ under two constant $\Delta K$ conditions. The crack propagated about $7.25 \mathrm{~mm}$ (including $2 \mathrm{~mm}$ precrack)

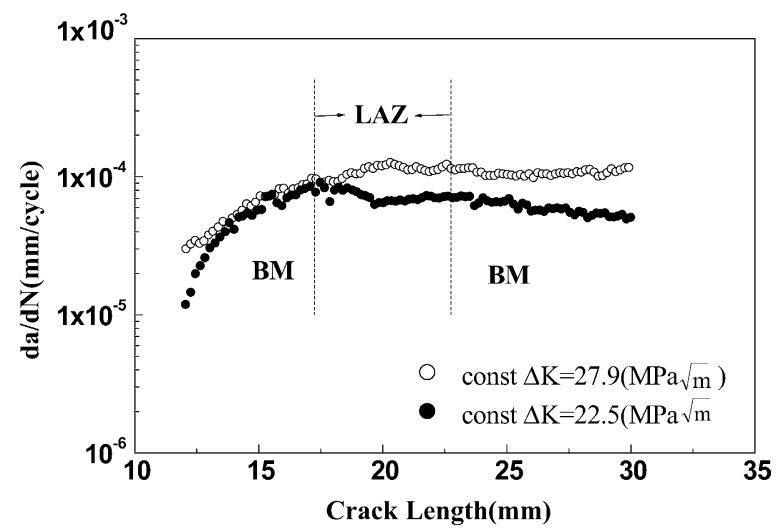

Fig. 5. Fatigue crack growth behaviour of NL-10 specimens tested under constant $\Delta K$ conditions. in the base metal (BM) before entering the LAZ with the width of approximately $5.0 \mathrm{~mm}$. Higher resistance to fatigue crack growth is found in the region ahead of LAZ, especially for the low $\Delta K$ condition. It indicates that the presence of residual compressive stresses ahead of crack tip causes the reduction of FCGR in front of LAZ for the laser-annealed specimen. Horikawa and Takada [2,7] reported that residual welding stresses have a great influence on both crack initiation and growth at low $\Delta K$ and/or $R$ ratio. Especially for the specimen tested under the lower constant $\Delta K$ condition, e.g. $\Delta K=22.5 \mathrm{MPa} \mathrm{m}^{1 / 2}$, the initial retardation of crack growth was very prominent as shown in Fig. 5. Additionally, the initially high Paris law gradient associated with excessive acceleration of crack growth ahead of LAZ was also observed. As the crack grew gradually into the specimen, continuous relaxation of residual stresses led to reduce its impact on the fatigue crack growth. Meanwhile, the initially high retardation of crack growth reduced in the high $\Delta K$ condition, e.g. $\Delta K=27.9 \mathrm{MPa} \mathrm{m}^{1 / 2}$. It indicates that the effect of residual thermal stresses to impede crack growth is not so effective in the high $\Delta K$ condition.

As the crack propagating into the LAZ, the fatigue crack growth behavior is similar to that of the base plate. It is found that the residual stress measured in the region ahead of crack tip is $248 \mathrm{MPa}$ in tension, and the residual stress at the location $10 \mathrm{~mm}$ in front of the crack tip is $140 \mathrm{MPa}$ in compression. Based on the previous finite element simulated result, the residual stress ahead of the crack tip was completely tensile stress as the crack propagates into the LAZ [15]. It is noted that the enhanced FCGR does not take place in the experiment. It indicates that a tensile stress field ahead of the crack tip has little effect on the FCGR of the ductile 304SS. Okamoto has pointed out that residual compressive stresses remarkably suppress the inner surface crack growth of a low carbon steel pipe, while residual tensile stresses do not accelerate the FCGR to a large extent [3]. It is consistent with the current investigation.

Fig. 6(a) shows the SEM fractograph illustrating typical transgranular dominated fatigue fractured surface of the 304SS base metal. The fractograph of steel plate is primarily comprised of transgranular fracture and a few flat facets. According to Gao et al. $[19,20]$, both twin and austenite grain boundaries serve an easy path for crack propagation. Additionally, the fracture path along twin boundaries results in flat facets on the fractured surface [20]. The high resistance to crack propagation of the NL-10 specimen under low $\Delta \mathrm{K}$ condition is observed in Fig. 3. Obvious rubbed marks are observed in Fig. 6(b) as indicated by arrows. The rubbed marks with flat surfaces must be caused by residual compressive stresses. A tensile stress field will not promote the formation of rubbed marks. Consequently, the presence of residual compressive stresses ahead of the crack tip is further demonstrated on the fatigue-fractured surface of NL-10 specimen. Accordingly, the amount of rubbed marks on the fatigue-fractured surface is greatly decreased as the $\Delta K$ increasing. For specimens with lower residual compressive stress ahead of the 

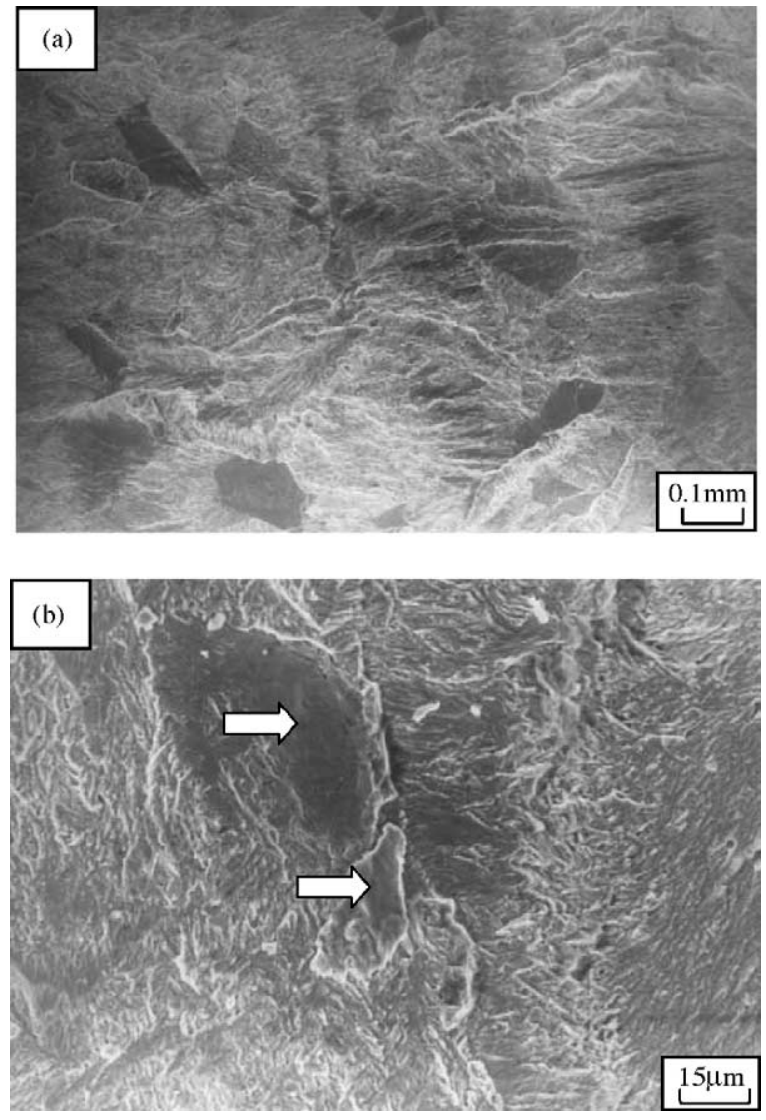

Fig. 6. The SEM fractographs of (a) the transgranular dominated fatigue fractured surface of the 304SS base metal and (b) a few rubbed marks on the NL-10 specimen.

crack tip, e.g. NL-SR and NL-5 specimens, the fatigue fracture appearance reveals nearly the same fracture morphology as that of base metal (Fig. 6(a)). Therefore, the less influence of the residual compressive stress for both NL-SR and NL-5 specimens is further confirmed by SEM fractographs.

\section{Summary}

The fatigue crack growth behavior of AISI 304 stainless steel (304SS) annealed by a $\mathrm{CO}_{2}$ laser was investigated in the study. For the laser-annealed 304SS specimen prior to introducing a notch, the high residual tensile stress is always located around the laser-annealed zone (LAZ). In the region away from the LAZ, the residual tensile stress is decreased and eventually converted from tensile into the compressive stress. A significant redistribution of the residual thermal stress is in progress after introducing a crack into the laser-annealed 304SS. For the specimen with the notch perpendicular to LAZ, the residual tensile stress is found in the LAZ, and the high residual compressive stress is also obtained in the region ahead of the notch tip. Consequently, the improved resistance to fatigue crack growth of the NL10 specimen preceding LAZ is attributed to the presence of residual compressive stress field ahead of the crack tip. In contrast, the NL-SR specimen behaves the similar FCGR as the base metal due to stress relief treatment of the laser-annealed specimen. The residual compressive stress enhances the effect of crack closure, and leads to the formation of rubbed marks on the fatigue-fractured surface. Whereas, the residual tensile stress field ahead of crack tip did not accelerate the crack growth as the fatigue crack propagates into the LAZ. It is concluded that a tensile stress field ahead of the crack tip has less influence on the FCGR of the ductile 304SS.

\section{Acknowledgement}

The authors gratefully acknowledge the financial support of the Republic of China National Science Council (contract number NSC 89-2216-E-019-001).

\section{References}

[1] D.W. Hammond, S.A. Menguid, Eng. Fract. Mech. 37 (1990) 373.

[2] K. Horikawa, Y. Takada, Trans. JWRI 12 (1984) 163.

[3] A. Okamoto, H. Nakamura, J. Press. Vess-T. ASME 112 (1990) 199.

[4] K.J. Kang, J.H. Song, Y.Y. Earmme, Fatigue Fract. Eng. Mater. Struct. 12 (1989) 363

[5] M. Beghini, L. Bertini, E. Vitale, Fatigue Fract. Eng. Mater. Struct. 17 (1994) 1433.

[6] Y.B. Lee, C.S. Chung, Y.K. Park, H.K. Kimt, Int. J. Fatigue 20 (1998) 565.

[7] K. Horikawa, A. Sakakibara, T. Mori, Trans. JWRI 12 (1983) 295.

[8] S. Fukuda, S. Watari, K. Horikawa, Trans. JWRI 8 (1979) 265.

[9] K.J. Kang, K. Song, Y.Y. Earmme, Fatigue, Fract. Eng. Mech. 13 (1990) 1.

[10] L. Bertini, Theor. Appl. Fract. Mech. 16 (1991) 135.

[11] M. Beghini, L. Bertini, Eng. Fract. Mech. 36 (1990) 379.

[12] W.J. Plumbridge, N. Knee, Scripta Metall. 19 (1985) 1029.

[13] S. Fukuda, Y. Tsuruta, Trans. JWRI 7 (1978) 67.

[14] A. Saxena, S.J. Hudak, Int. J. Fract. 14 (1978) 453.

[15] R.K. Shiue, C.T. Chang, M.C. Young, L.W. Tsay, Mater. Sci. Eng. A 364 (2004) 102.

[16] M. Parry, H. Nordberg, R.W. Hertzberg, Weld. Res. Suppl. 51 (1972) 485.

[17] S.J. Gill, D.W. Moon, E.A. Metzbower, T.W. Crooker, Weld. Res Suppl. 65 (1986) 48.

[18] L.W. Tsay, C.C. Liu, Y.H. Chao, Y.H. Shieh, Mater. Sci. Eng. A 299 (2001) 16.

[19] M. Gao, S. Chen, R.P. Wei, Metall. Trans. 23A (1992) 355.

[20] M. Gao, R.P. Wei, Scripta Metall. 26 (1992) 1175. 